\title{
GELFAND-KIRILLOV DIMENSION OF RINGS OF FORMAL DIFFERENTIAL OPERATORS ON AFFINE VARIETIES
}

\author{
S. P. SMITH
}

\begin{abstract}
Let $A$ be the coordinate ring of a smooth affine algebraic variety defined over a field $k$. Let $D$ be the module of $k$-linear derivations on $A$ and form $A[D]$, the ring of differential operators on $A$, as follows: consider $A$ and $D$ as subspaces of End $_{h} A$ ( $A$ acting by left multiplication on itself), and define $A[D]$ to be the subalgebra generated by $A$ and $D$. Let rk $D$ denote the torsion-free rank of $D$ (that is, rk $D=\operatorname{dim}_{F} F \otimes_{A} D$ where $F$ is the quotient field of $A$ ). The ring $A[D]$ is a finitely generated $k$-algebra so its Gelfand-Kirillov dimension $\operatorname{GK}(A[D])$ may be defined. The following is proved.

Theorem. GK( $A[D])=\operatorname{tr} \operatorname{deg}_{h} A+$ rk $D=2 \operatorname{tr} \operatorname{deg}_{h} A$.

Actually we work in a more general setting than that just described, and although a more general result is obtained, this is the most natural and important application of the main theorem.
\end{abstract}

1. Introduction. Let $A$ be a finitely generated commutative algebra over the field $k$. In the terminology of [9] let $D$ be a $(k, A)$-Lie algebra. We recall the definition.

(i) $D$ is a Lie algebra over $k$, with the Lie product denoted [, ];

(ii) $D$ is an $A$-module;

(iii) there is an $A$-module homomorphism, $\theta: D \rightarrow \operatorname{Der} A$ (the module of $k$-linear derivations on $A$ ); we denote $\theta(d)(a)$ by $d(a)$ for $d \in D, a \in A$;

(iv) these structures are related by the requirement that $\left[d_{1}, a d_{2}\right]=a\left[d_{1}, d_{2}\right]+$ $d_{1}(a) d_{2}$ for $d_{1}, d_{2} \in D$ and $a \in A$.

The most natural examples of $(k, A)$-Lie algebras are simply submodules $D$ of Der $A$, which are closed under the Lie bracket on Der $A$.

Given a $(k, A)$-Lie algebra $D$ we form the ring of formal differential operators, $A\langle D\rangle$, as follows: it is the factor ring, $T_{A}(D) / J$, of the tensor algebra of the $A$-module $D$, by the ideal $J$ generated by the relations $d a-a d=d(a)$ for all $a \in A$, $d \in D$ and $d_{1} d_{2}-d_{2} d_{1}=\left[d_{1}, d_{2}\right]$ (for all $d_{1}, d_{2} \in D$ ). When $D$ is an abelian Lie algebra and a finitely generated free $A$-module the ring $A\langle D\rangle$ has been studied by a number of authors [1], [3-5].

We shall always assume $D$ is a finitely generated $A$-module, in which case $A\langle D\rangle$, being a factor of $T_{A}(D)$, is a finitely generated $k$-algebra. Thus the Gelfand-Kirillov

Received by the editors October 15, 1982 and, in revised form, April 11, 1983.

1980 Mathematics Subject Classification. Primary 16A55; Secondary 16A56, 16A72, 17B40.

Key words and phrases. Gelfand-Kirillov dimension, differential operators, derivations.

c.1984 American Mathematical Society $0002-9939 / 84 \$ 1.00+\$ .25$ per page 
dimension of $A\langle D\rangle$ may be defined to be

$$
\mathrm{GK}(A\langle D\rangle)=\limsup _{n \rightarrow \infty}\left(\log \operatorname{dim} W^{n} / \log n\right)
$$

where $W$ is a finite-dimensional subspace of $A\langle D\rangle$ generating $A\langle D\rangle$ as a $k$-algebra. We adopt the convention that any subspace generating a $k$-algebra actually contains the field $k ; W^{n}$ denotes the linear span of all words $w_{1} \cdots w_{n}$ with each $w_{j} \in W$.

For any integral domain $C$ with quotient field $F$, and any finitely generated $C$-module $M$, define the rank of $M$ by rk $M=\operatorname{dim}_{F} F \otimes_{C} M$.

The problem is to determine $\operatorname{GK}(A\langle D\rangle)$ in terms of other invariants of $A$ and $D$. We prove

Theorem A. If $D$ is a projective A-module and $k$ is of characteristic 0 , then

$$
\begin{aligned}
\mathrm{GK}(A\langle D\rangle) & =\max \left\{\operatorname{tr} \operatorname{deg}_{k}(A / P)+\mathrm{rk}_{A / P}(D / P D) \mid P \text { is a minimal prime of } A\right\} \\
& =\operatorname{tr} \operatorname{deg}_{k} S_{A}(D),
\end{aligned}
$$

where $S_{4}(D)$ is the symmetric algebra of $D$.

The statement of the theorem simplifies when $A$ is a domain or $D$ is free: if $A$ is a domain the assumption on the characteristic of $k$ is unnecessary and $\operatorname{GK}(A\langle D\rangle)=$ $\operatorname{tr} \operatorname{deg} A+\operatorname{rk} D$; if $D$ is free then $\mathrm{GK}(A\langle D\rangle)=\operatorname{tr} \operatorname{deg} A+\operatorname{rk} D$. When $D$ is free and the image of the generators of $D$ in $\operatorname{Der} A$ span a finite dimensional solvable Lie algebra it follows easily from [8] that $\mathrm{GK}(A\langle D\rangle)=\operatorname{tr} \operatorname{deg} A+\operatorname{rk} D$.

An earlier version of this paper benefitted from the referee's criticism and we would like to express our thanks.

2. Generalities concerning $A\langle D\rangle$. The natural map $A \oplus D \rightarrow A\langle D\rangle$ is an embedding and we identify $A$ and $D$ with their images in $A\langle D\rangle$. The ring $A\langle D\rangle$ is endowed with a natural filtration by the subspaces $R_{n}=(A+D)^{n}=A+D+D^{2}$ $+\cdots+D^{n}$. Using the Lie bracket on $D$ it is easy to check that if $x \in R_{n}$ and $y \in R_{m}$ then $x y-y x \in R_{n+m-1}$. From this it follows that the associated graded algebra gr $A\langle D\rangle=\sum_{n=0}^{\infty} R_{n} / R_{n-1}$ is commutative. Furthermore, gr $A\langle D\rangle$ is generated as an algebra over $A$ by the finitely generated $A$-module $R_{1} / R_{0} \cong D$, so there is a canonical surjection $S_{A}(D) \rightarrow \operatorname{gr} A\langle D\rangle$, where $S_{A}(D)$ is the symmetric algebra of the $A$-module $D$.

Proposition 2.1 [9. Theorem 3.1]. If $D$ is projective, the canonical map $S_{A}(D) \rightarrow$ gr $A\langle D\rangle$ is an isomorphism.

Proof. Just observe that $A\langle D\rangle$ coincides with the ring $V(A, D)$ of [9] (or the ring $V_{4}$ of [7]).

Recall the definition [6] of the ring $D_{X}$ of differential operators on an affine algebraic variety $X$.

Proposition 2.2 [11]. Let $X$ be a smooth affine algebraic variety with coordinate ring $A$. Then $\operatorname{Der} A$ is a projective $A$-module and $D_{X}$ coincides with $A\langle\operatorname{Der} A\rangle$. Furthermore, the filtration on $D_{X}$ given $b y$ the order of the differential operators coincides with the filtration on $A\langle\operatorname{Der} A\rangle$ given $b y$ the powers of the subspace $A+\operatorname{Der} A$. 
Proof. By [11, Theorem 18.2], as $X$ is smooth, $D_{X}$ is the subalgebra of End $_{h} A$ generated by $A$ and $\operatorname{Der}_{h} A$, so there is a natural surjection $\phi: A\langle\operatorname{Der} A\rangle \rightarrow D_{X}$ and $\phi$ preserves the filtration. Hence, $\phi$ induces a map $\operatorname{gr} \phi: \operatorname{gr} A\langle\operatorname{Der} A\rangle \rightarrow \operatorname{gr} D_{\chi}$. But by Proposition 2.1, gr $A\langle\operatorname{Der} A\rangle \cong S_{A}(\operatorname{Der} A)$ and by [11, Theorem 18.2], gr $D_{X} \cong$ $S_{A}(\operatorname{Der} A)$ also. Hence $\mathrm{gr} \phi$ is an isomorphism. The commutativity of the diagram

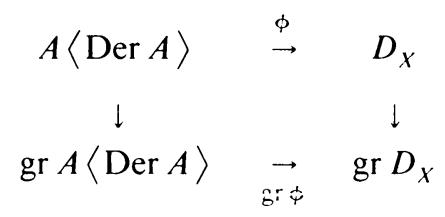

(where the vertical maps are the gradings) ensures that $\phi$ is injective and hence an isomorphism.

COROllaRY 2.3. If $X$ is a smooth affine algebraic variety then $\operatorname{GK}\left(D_{X}\right)=2 \operatorname{dim} X$.

Proof. Just apply Theorem A, together with the standard fact that $\operatorname{tr} \operatorname{deg}_{k} A=$ rk $\operatorname{Der} A=\operatorname{dim} X$.

In [2, Chapter 2, §6] Bjork considers a noncommutative ring $R$ equipped with a filtration such that the associated graded algebra is commutative and noetherian. An integer $d(R)$ is defined in terms of the properties of the associated graded algebra. The ring $A\langle D\rangle$ fits into this context and once Theorem $\mathrm{A}$ has been established it is easy to obtain the following

COROllary 2.4. If $D$ is projective, finitely generated and char $k=0$, then $\mathrm{GK}(A\langle D\rangle)=d(A\langle D\rangle)$.

Proof. The definition of $d$ ensures that $d(A\langle D\rangle)$ equals the classical Krull dimension of $\operatorname{gr} A\langle D\rangle$. But $\operatorname{gr} A\langle D\rangle \cong S_{A}(D)$ by Proposition 2.1 and hence $d(A\langle D\rangle)=\operatorname{tr} \operatorname{deg} S_{A}(D)=\mathrm{GK}(A\langle D\rangle)$ by Theorem A.

We do not know if $d(M)$ and $\mathrm{GK}(M)$ coincide for an arbitrary finitely generated $A\langle D\rangle$-module $M$. In the special case when $A\langle D\rangle$ is a Weyl algebra, Bjork [2. Chapter 3, §A.2.5] show that $d(M)=\mathrm{GK}(M)$ for all finitely generated $M$.

3. Two lemmas concerning polynomials. The following is useful in the context of Hilbert polynomials.

Lemma 3.1. Let $f \in \mathbf{Q}[x]$. Then $f$ is a polynomial of degree $d$ if and only if the polynomial $\tilde{f}$, defined by $\tilde{f}(x)=f(x+1)-f(x)$, is a polynomial of degree $d-1$.

We will need another lemma concerning polynomials.

Lemma 3.2. Let $p, q \in \mathbf{Q}[x]$ be polynomials of degree $r$, $t$, respectively, and suppose $c \in \mathbf{N}$ is fixed. Define a function $f$ on $\mathbf{N}$ by $f(n)=p(0) q(n)+p(1) q(n-1)$ $+\cdots+p(n-c) q(c)$. Then $f$ is a polynomial of degree $r+t+1$.

Proof. By induction on the degree of $q$. If $\operatorname{deg} q=0$ then $q$ is a constant; say $q(n)=Q$. Thus, we have $f(n)=Q(p(0)+\cdots+p(n-c))$ and $f(n+1)-f(n)=$ $Q p(n+1-c)$. This is a polynomial of degree $r$. so by Lemma 3.1, $f$ is a polynomial of degree $r+1$. 
Suppose now that the result is true for polynomials of degree $t-1$ and that $\operatorname{deg} q=t$. Then

$$
f(n+1)-f(n)=\sum_{i=0}^{n-c} p(i)[q(n+1-i)-q(n-i)]+p(n+1-c) q(c) .
$$

The polynomial $q(n+1-i)-q(n-i)$ is of degree $t-1$, so the induction hypothesis applied to the function $g(n)=\sum_{i=0}^{n-c} p(i)[q(n+1-i)-q(n-i)]$ ensures that $g$ is of degree $r+t$. As $p(n+1-c) q(c)$ is of degree $r, f(n+1)-f(n)$ is a polynomial of degree $r+t$. Another application of Lemma 3.1 shows $f(n)$ is of degree $r+t+1$.

4. Normal form of elements in $A\langle D\rangle$. Let $C$ be a finite-dimensional generating subspace of $D$ with basis $d_{1}, \ldots, d_{r}$. Choose a finite-dimensional generating subspace $V$ of $A$ with the property that $[C, C] \subset V C$ and $C(V) \subset V^{2}$. It is possible to find such a subspace: just pick any subspace $U$ of $A$, generating $A$ and satisfying $[C, C] \subset U C$; for some $l, C(U) \subset U^{l}$, hence $C\left(U^{l}\right) \subset U^{2 l}$, and putting $V=U^{l}$ we have a subspace with the required properties.

Notice that $C\left(V^{n}\right) \subset \sum_{j=0}^{n-1} V^{j} C(V) V^{n-j-1} \subset V^{n+1}$ for all $n$. Another way of expressing this is that $C V^{n} \subset V^{n} C+V^{n+1}$; we shall make frequent use of this fact.

Let $C_{n}$ denote the $k$-linear span of $\left\{d_{1}^{i_{1}} \cdots d_{r}^{i_{r}} \mid i_{1}+\cdots+i_{r}=n\right\}$ with the convention that $C_{0}=k$; notice that $C=C_{1}$. It is clear that $R_{n}=A C_{0}+A C_{1}+\cdots+A C_{n}$; if an element of $A\langle D\rangle$ is written in the form

$$
\sum a_{i_{1} \cdots i_{r}} d_{1}^{i_{1}} \cdots d_{r}^{i_{r}} \quad\left(\text { where each } a_{i_{1} \cdots i_{r}} \in A\right),
$$

we shall say that the element is expressed in normal form.

Put $W=V \oplus C ; W$ is a finite-dimensional generating subspace of $A\langle D\rangle$. It is necessary to study $\operatorname{dim} W^{n}$ and as a first step towards this we will show that $W^{n}=\sum_{t=0}^{n} V^{n-t} C_{t}$ (Theorem 4.4). This result may be thought of as a statement about the normal form of elements in $W^{n}$, or as a statement about the product of two elements in normal form.

LeMma 4.1. $C^{p} V \subset \sum_{j=0}^{p} V^{j+1} C^{p-j}$.

Proof. It is true for $p=1$ by what has already been said. Suppose that the statement is true for $p$. We shall prove it true for $p+1$ :

$$
\begin{aligned}
C^{p+1} V & \subset \sum_{j=0}^{p} C V^{j+1} C^{p-j} \subset \sum_{j=0}^{p}\left(V^{j+1} C+V^{j+2}\right) C^{p-j} \\
& \subset \sum_{j=0}^{p} V^{j+1} C^{p-j+1}+V^{j+2} C^{p-j} \subset \sum_{j=0}^{p+1} V^{j+1} C^{p-j+1} .
\end{aligned}
$$

LeMma 4.2. $\left[C, C_{m}\right] \subset \sum_{j=0}^{m-1} V^{j+1} C^{m-j}$.

Proof. Because $C_{m} \subset C^{m}$ we have

$$
\left[C, C_{m}\right] \subset\left[C, C^{m}\right] \subset \sum_{i=0}^{m-1} C^{i}[C, C] C^{m-i-1} .
$$


Hence $\left[C, C_{m}\right] \subset \sum_{i=0}^{m-1} C^{i} V C^{m-i}$ and the result follows at once from Lemma 4.1 applied to the term $C^{i} V$.

LeMma 4.3. $C^{p} \subset \sum_{j=0}^{p-1} V^{j} C_{p-j}$.

Proof. The result is true for $p=1$. Suppose it is true for all integers less than or equal to $p$. Then

$$
C^{p+1} \subset \sum_{j=0}^{p-1} C V^{j} C_{p-j} \subset \sum_{j=0}^{p-1}\left(V^{j} C+V^{j+1}\right) C_{p-j} .
$$

We claim that for $m \leqslant p, C C_{m} \subset \sum_{i=0}^{m} V^{i} C_{m+1-i}$. This is true for $m=0$, and we prove it by induction on $m$. Suppose it is true for $m-1$. Let $d_{j+1}$ be one of the basis elements of $C$, and pick $d_{r}^{i_{1}} \cdots d_{r}^{i_{r}} \in C_{m}$. It is enough to show that

$$
x=d_{j+1}\left(d_{1}^{i_{1}} \cdots d_{r}^{i_{r}}\right) \in \sum_{i=0}^{m} V^{i} C_{m+1-i} .
$$

Now

$$
d_{j+1}\left(d_{1}^{i_{1}} \cdots d_{r}^{i_{r}}\right)=\left(\left[d_{j+1}, d_{1}^{i_{1}} \cdots d_{j}^{i_{j}}\right]-d_{1}^{i_{1}} \cdots d_{j}^{i_{j}} d_{j+1}\right) d_{j+1}^{i_{j+1}} \cdots d_{r}^{i_{r}}
$$

is an element of $\left[C, C_{t}\right] C_{m-t}+C_{m+1}$ for some $t, m \geqslant t \geqslant 1$. By Lemma 4.2 we see that

$$
\begin{aligned}
x & \in \sum_{i=0}^{t-1} V^{i+1} C^{t-i} C_{m-t}+C_{m+1} \subset \sum_{i=0}^{t-1} V^{i+1} C^{m-i}+C_{m+1} \\
& \subset \sum_{i=0}^{m-1} V^{i+1} C^{m-i}+C_{m+1} .
\end{aligned}
$$

If we now apply the induction hypothesis of the lemma to $C^{m-i}$ for each $i$ $(0 \leqslant i \leqslant m-1)$, we have that

$$
\begin{aligned}
x & \in \sum_{i=0}^{m-1} V^{i+1}\left(\sum_{j=0}^{m-i-1} V^{j} C_{m-i-j}\right)+C_{m+1} \\
& =\sum_{i=0}^{m-1} \sum_{j=0}^{m-i-1} V^{i+j+1} C_{m-i-j}+C_{m+1} .
\end{aligned}
$$

It is now clear that the claim is true for $m$.

Returning to the lemma, and applying the claim we have established that

$$
C^{p+1} \subset\left(\sum_{j=0}^{p-1} V^{j} \sum_{i=0}^{p-j} V^{i} C_{p-j+1-i}\right)+\sum_{j=0}^{p-1} V^{j+1} C_{p-j} .
$$

The truth of the lemma for $p+1$ follows.

THEOREM 4.4. $W^{n}=\sum_{t=0}^{n} V^{n-t} C_{t}$.

Proof. We proceed by induction, the case $n=1$ being true from the definition of $W$. It is clear that $W^{n} \supset \sum_{t=0}^{n} V^{n-t} C_{t}$ as $C_{t} \subset W^{t}$, and $V^{n-t} \subset W^{n-t}$. We prove the 
reverse inclusion. Suppose it is true for $n$. Then $W^{n+1}=\sum_{t=0}^{n} V^{n+1-t} C_{t}+$ $\sum_{t=0}^{n} C V^{n-t} C_{t}$. The first of these terms belongs to $\sum_{t=0}^{n+1} V^{n+1-t} C_{t}$, and it remains only to prove that the second also does. By Lemma 4.3, and the fact that $C C_{t} \subset C^{t+1}$ we have

$$
\begin{aligned}
\sum_{t=0}^{n} C V^{n-t} C_{t} & \subset \sum_{t=0}^{n}\left(V^{n-t} C+V^{n-t+1}\right) C_{t} \\
& \subset \sum_{t=0}^{n} V^{n-t}\left(\sum_{j=0}^{t} V^{j} C_{t+1-j}\right)+\sum_{t=0}^{n+1} V^{n+1-t} C_{t} \\
& \subset \sum_{t=0}^{n+1} V^{n+1-t} C_{t} .
\end{aligned}
$$

\section{Proof of the theorem.}

LeMma 5.1. If $\operatorname{gr} A\langle D\rangle \cong S_{A}(D)$ then $\operatorname{GK}(A\langle D\rangle) \geqslant \operatorname{GK}\left(S_{A}(D)\right)$.

Proof. Pick $V, C, W$ as in $\S 4$, so that $W^{n}=\sum_{t=0}^{n} V^{n-t} C_{t}$. Now $S_{A}(D)$ is generated as a $k$-algebra by the subspace $V+\bar{C}$, where $\bar{C}=C+R_{0} / R_{0}$. So to evaluate $\mathrm{GK}\left(S_{A}(D)\right)$ we must examine $\operatorname{dim}(V+\bar{C})^{n}$. But

$$
\begin{aligned}
\operatorname{dim}(V+\bar{C})^{n} & =\operatorname{dim} \sum_{i=0}^{n} V^{n-t}(\bar{C})^{t}=\operatorname{dim} \sum_{t=0}^{n} V^{n-t}\left(C_{t}+R_{t-1} / R_{t-1}\right) \\
& =\operatorname{dim} \sum_{t=0}^{n}\left(V^{n-t} C_{t}+R_{t-1} / R_{t-1}\right) \\
& \leqslant \operatorname{dim} \sum_{t=0}^{n} V^{n-t} C_{t}=\operatorname{dim} W^{n}
\end{aligned}
$$

(by Theorem 4.4), and the lemma follows from the inequality $\operatorname{dim}(V+\bar{C})^{n} \leqslant$ $\operatorname{dim} W^{n}$.

We shall first obtain $\mathrm{GK}(A\langle D\rangle)$ under the assumption that $A$ is a domain. The general case will be reduced to the domain case by considering $A / P$ for the minimal primes $P$ of $A$.

LEMma 5.2. If $A$ is a domain and $D$ a torsion-free $A$-module then

$$
\mathrm{GK}\left(S_{A}(D)\right)=\operatorname{tr} \operatorname{deg}_{k} A+\operatorname{rk} D .
$$

Proof. As remarked in [10], under the hypotheses of the lemma, $S_{A}(D)$ is a torsion-free $A$-module; in particular, regular elements of $A$ remain regular as elements of $S_{A}(D)$. Hence, the natural map $S_{A}(D) \rightarrow F \otimes_{A} S_{A}(D)$ is an embedding and the latter may be considered as lying in the quotient field of $S_{A}(D)$. In a commutative ring the GK-dimension coincides with the transcendence degree, so $\mathrm{GK}\left(S_{A}(D)\right)=\operatorname{tr} \operatorname{deg}_{k}\left(F \otimes_{A} S_{A}(D)\right)$. It is standard that $F \otimes_{A} S_{A}(D) \cong S_{F}\left(F \otimes_{A} D\right)$; but $F \otimes_{A} D$ is just a free $F$-module on $r=$ rk $D$ generators, so $S_{F}\left(F \otimes_{A} D\right) \cong$ $F\left[X_{1}, \ldots, X_{r}\right]$, the polynomial extension in $r$ indeterminates. The transcendence degree of $F\left[X_{1}, \ldots, X_{r}\right]$ is simply $\operatorname{tr} \operatorname{deg}_{k} F+r$; whence the result. 
Combining these two lemmas gives half of Theorem A (at least for $A$ a domain) as any projective module is certainly torsion free.

Proposition 5.3. If $A$ is a domain and $D$ torsion free, then

$$
\mathrm{GK}(A\langle D\rangle) \leqslant \operatorname{tr} \operatorname{deg} A+\operatorname{rk} D .
$$

Proof. Pick $0 \neq x \in A$ such that, if $B=A\left[x^{-1}\right]$ then $E=B \otimes_{A} D$ is a free $B$-module of rank $r=$ rk $D$. As $D$ is torsion free, so too is $S_{A}(D)$, and hence $A\langle D\rangle$ itself is a torsion-free $A$-module. In particular, $x$ is a regular element of $A\langle D\rangle$ so the natural map $A\langle D\rangle \rightarrow B\langle E\rangle$ is injective, and it is enough to prove the proposition for $B\langle E\rangle$. So assume $D$ is a free $A$-module.

Pick $W, V, C$ as in $\S 4$, but with the extra condition that $C$ is a vector space of dimension $r=\operatorname{rk} D$. As $\operatorname{gr} A\langle D\rangle \cong S_{A}(D) \cong A\left[X_{1}, \ldots, X_{r}\right]$, one has for all $t$ that $\operatorname{dim} C_{t}=\left(\begin{array}{c}t+r \\ r\end{array}\right)$. Because $W^{n}=\sum_{t=0}^{n} V^{n-t} C_{t}$,

$$
\operatorname{dim} W^{n} \leqslant \sum_{t=0}^{n}\left(\operatorname{dim} V^{n-t}\right)\left(\operatorname{dim} C_{t}\right)=\sum_{t=0}^{n} q(n-t) p(t),
$$

say, where $q(n-t)=\operatorname{dim} V^{n-t}, p(t)=\operatorname{dim} C_{t}$. However, $p(t)$ is a polynomial of degree $r$, and $q(n-t)$ is a polynomial of degree $d=\operatorname{tr} \operatorname{deg} A$. Hence by Lemma 3.2, $\sum_{t=0}^{n} q(n-t) p(t)$ is a polynomial of degree $d+r$. Because $\operatorname{dim} W^{n}$ is bounded above by a polynomial of $\operatorname{degree} \operatorname{tr} \operatorname{deg} A=\operatorname{rk} D$, the result follows.

Now we have the upper bound for $\operatorname{GK}(A\langle D\rangle)$, and combining the previous three lemmas proves Theorem A for $A$ a domain. Notice that to prove the theorem for a domain no assumption on char $\dot{k}$ was required. The necessity of the condition becomes clear in the following (where we no longer assume $A$ is a domain).

Proposition 5.4. Let $A$ be an algebra over a field of characteristic zero. Let $D$ be a projective $(k, A)$-Lie algebra. Then

$$
\mathrm{GK}(A\langle D\rangle)=\max \left\{\mathrm{GK}\left(\frac{A}{P}\langle D / D P\rangle\right) \mid P \text { is a minimal prime of } A\right\} .
$$

Proof. When $A$ is an algebra over a field of characteristic zero then the minimal primes of $A$ are invariant under every derivation on $A$. There are minimal primes $P_{1}, \ldots, P_{n}$ of $A$ with $P_{1} \cdots P_{n}=0$. Putting $R=A\langle D\rangle$ and using the fact that $R P_{i}=P_{i} R$ is an ideal of $R$, one has the product $\left(P_{1} R\right)\left(P_{2} R\right) \cdots\left(P_{n} R\right)$ equal to zero. Consequently, $\mathrm{GK}(A\langle D\rangle)=\max \left\{\mathrm{GK}\left(R / P_{i} R\right) \mid i=1, \ldots, n\right\}$. However, given an ideal $I$ of $A$, invariant under any derivation, $R / I R \cong(A / I)\langle D / D I\rangle$. This follows from the fact that the diagram

$$
\begin{array}{ccc}
A\langle D\rangle & \rightarrow & (A / I)\langle D / D I\rangle \\
\downarrow & & \downarrow \\
S_{A}(D) & \rightarrow & S_{A / I}(D / D I)
\end{array}
$$

(with the vertical maps being the gradings and the horizontal maps being those induced by the natural surjections $A \rightarrow A / I, D \rightarrow D / D I)$ is commutative, and the kernel of the lower map is the ideal of $S_{A}(D)$ generated by $I$. 
Corollary 5.5. Let $A$ and $D$ be as above. Then

$\mathrm{GK}(A\langle D\rangle)=\max \left\{\operatorname{tr} \operatorname{deg}_{k} A / P+\mathrm{rk}_{A / P}(D / D P) \mid P\right.$ is a minimal prime of $\left.A\right\}$.

Proof. Just use the above propositions, and note that if $D$ is projective as an $A$-module then $D / D P$ is projective as an $A / P$-module. This completes the proof of Theorem A.

\section{REFERENCES}

1. J. E. Bjork, The glohal homological dimension of some algebras of differential operators, Invent. Math. 17 (1972), 67-78.

2. Rings of differential operators, North-Holland Math. Library, North Holland, New York. 1979.

3. K. R. Goodearl, Glohal dimension of differential operator rings, Proc. Amer. Math. Soc. 45 (1974), $315-322$.

4. Global dimension of differential operator rings. II, Trans. Amer. Math. Soc. 209 (1975), $65-85$.

5. . Glohal dimension of differential operator rings. III, J. London Math. Soc. 17 (1978), 397-409.

6. A. Grothendieck, Eléments de géométrique algébrique. IV, Inst. Hautes Études Sci. Publ. Math. No. 32 (1967)

7. G. Hochschild, B. Kostant and A. Rosenberg, Differential forms on regular affine algebras, Trans. Amer. Math. Soc. 102 (1962), 383-408.

8. M. Lorenz, On the Gelfand-Kirillow dimension of skew polynomial rings, preprint.

9. G. S. Rinehart, Differential forms on general commutative algebras, Trans. Amer. Math. Soc. 108 (1963), 195-222.

10. P. Samuel, Anneaux gradues factoriels et modules reflexifs, Bull. Soc. Math. France 92 (1964), 234-249.

11. M. Sweedler, Groups of simple algebras, Inst. Hautes Études Sci. Publ. Math. 44 (1974).

Department of Mathematics, University of Southern California, los Angeles, California 90089

Current address: Mathematics Institute, University of Warwick, Coventry CV4 7AL, England 